\title{
Current Candidate Genes as Biomarkers for Major Depressive Disorders: A Review of Current Approaches and Findings With Respect to Ethnicity
}

\author{
Shanta Nair ${ }^{\mathrm{a}}$, C.H. Nair ${ }^{\mathrm{b}}$ and Shabbir Moochhala, \\ ${ }^{a}$ Macquarie University, Department of Biology, Balaclava Road, North Ryde NSW 2109, Australia \\ ${ }^{b}$ PrIME Biologics Pte Ltd, 41 Science Park Road \#01-04/11 The Gemini (Lobby A), 117610, Singapore \\ ${ }^{c}$ Department of Pharmacology, Yong Loo Lin School of Medicine, Clinical Research Centre \#05-09, 10 Medical Drive, \\ 117597, Singapore
}

\begin{abstract}
Major Depressive Disorder (MDD) currently affects people worldwide. Despite its multi-faceted origins and symptoms, and its myriad manifestations and outcomes, work is underway to understand the root causes of the disorder. Genetic studies, in particular, have focused on finding candidate genes for MDD, and investigating links between these genes and any particular group of people. It is hoped that these studies may shed light on the types of people that are commonly affected, and what factors make a person more or less vulnerable to depressive disorders. Multiple factors have been considered, including socioeconomic status, urban versus rural environments, ethnicity, lifestyle factors such as substance abuse and both pre- and post-natal early traumas. Through various methods, including network analyses and bioinformatics among others, significant links have been found between socioeconomic status, urban living, and MDD. Moreover, epigenetic links have been discovered between the prevalence of MDD and the prevalence of other diseases such as cardiovascular disease and other forms of mental illness, making it clear that environment plays a key role in overall health and wellbeing. While no statistically significant link between ethnicity and MDD has been found in candidate genes thus far despite certain ethnicity-based polymorphisms influencing clinical outcomes with different treatments, it is much more apparent that urban environments and early stress contribute to the prevalence of MDD.
\end{abstract}

Keywords: Biomarkers, candidate genes, depression, FKBP5, MDD, Polymorphisms.

\section{INTRODUCTION}

Major Depressive Disorder, or MDD, is a heterogeneous mental disorder commonly characterised by multiple levels of mood-affected symptoms [1]. It is most commonly triggered by past traumatic events [2, 3]. Globally, MDD has a documented lifetime prevalence of $9-19 \%$, with a significant heritability factor of $37-43 \%$ [4], and with women being generally more prone to depressive disorders than men [5]. This suggests that the susceptibility of a person to developing MDD has a basis in the inherited genome as well as in the environment. Current research seeks to reconcile environmental traumas and stresses as visible causes of MDD with the theorized heritable genetic causes. A multifaceted link between genetic expression and MDD is also well-supported by the current literature [6]. As many as 151 candidate genes whose variants have been associated with the development of MDD have been documented to date [7]. However, finding the exact number of causal genes, if in fact there is a concrete set of causal genes, has proved difficult so far, as the disease is well known to manifest itself differently from person to person, with different symptoms and severity.

*Address correspondence to this author at the Department of Pharmacology, Yong Loo Lin School of Medicine, Clinical Research Centre \#05-09, 10 Medical Drive, Singapore 117597; Tel: 65 4857201: Fax: 65485 7226;

E-mail: mshabbir@dso.org.sg
However, the recent completion of the human genome map and recent advances in bioinformatics, computing power, and statistical analysis have allowed researchers to further examine this issue. For the first time the combination of techniques such as microarray analysis [8] and systems network analysis [7] allows the compilation of candidate genes and their resulting cascades, and allows further comparison and contrast between the effects of variants of each gene on the population's mental health. Finding causal links between gene variation, the environment, and major depressive disorder may allow the development of personalised gene therapies to improve overall mental health, as well as preventative measures to decrease the overall likelihood and prevalence of the disease. MDD has also been seen to affect the entire body, not just a patient's psychology [9], making preventative measures and new treatments essential to improving the overall health and wellbeing of the public and reducing the prevalence of other common diseases, such as cardiovascular failure and anxiety disorders.

So far, the current research is seeking answers to a set of questions put forward by these findings that will hopefully shed some light on the rather tangled nature of this disease:

- How many candidate genes are there linked to MDD? 


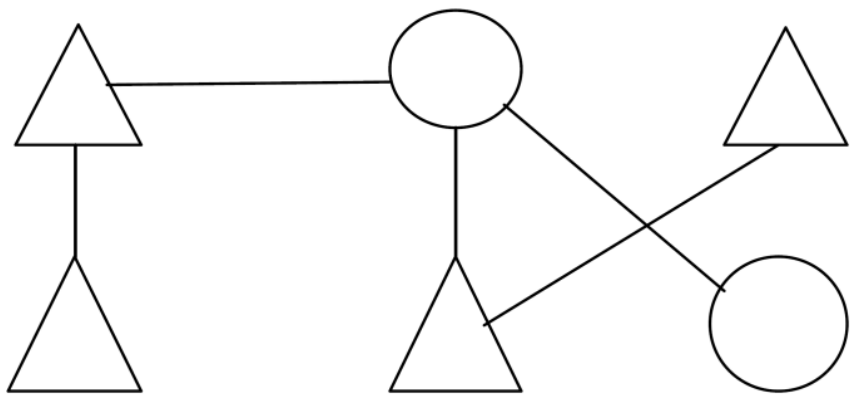

Fig. (1). An example of a network map of MDD-specific gene interactions.

- Which, if any, are linked with more statistical strength to the disease?

- Are there any causal links to be found with the rest of the genome or the environment, such as ethnicity or social status?

This paper will review the current major research being carried out in this field, and give a comprehensive overview of current findings, while examining the existing data for any apparent links between the variants of MDD candidate genes and ethnic grouping.

\section{PREVIOUS RESEARCH APPROACHES AND THEIR FINDINGS}

Several different analytical approaches have been applied to the question of finding and mapping the effects of candidate genes, each with varying amounts of success.

One of the most successful and useful tools for visualising the current state of progress in the field has been the network systems approach. Using advances in bioinformatics, the interactions and results of candidate genes have been visualised and mapped using an approach similar to mapping commands and actions in a computing system [7]. Using this networked map, we can now visualise the field of currently known candidate genes, as well as their interactions with other genes, their position in any gene cascades, and their effects on the prevalence and development of MDD. Fig. (1) illustrates this principle: in a simple diagram, triangles indicate known MDD candidate genes, while circles indicate associated genes. Interconnecting lines represent gene cascades, or associated genes that in turn trigger the expression of MDD genes.

As illustrated in Fig. (1), this presents a difficulty in the current quest to find and map MDD candidate genes. As mentioned earlier, there are as many as 151 candidate genes currently documented, and each of them has been found to be involved in a series of associations, cascades and links much like the diagram above. However, the sheer number of candidate genes and all their associated and cascaded genes makes the above diagram pale in comparison-- in reality, the web of genes, links, and potential outcomes is staggeringly convoluted [7]. The key in this research field, therefore, lies in unravelling the web and tracing groups of candidate genes back to the factors that dictate their expression. Herein lies the key to this research, and the thesis of this review: that it is not merely the genome, but also the environment that dictates the development of MDD. This illustrates the need for a multifaceted approach to the problem, incorporating several different research methods to help untangle this knot of candidate genes and causal factors.

Another approach commonly being used in this field as an analytical tool is microarray analysis, paired with statistical meta-analysis, as used by Wang et al. in their 2012 paper. Using these two approaches in conjunction has proved useful in eliminating or reducing the effect of confounding variables when examining candidate genes. That is, since genes may be expressed under many different conditions and cause different effects, the resulting data may be confusing and inconclusive. A meta-analysis is therefore used to reduce the amount of "background noise" in the data and get to the root of each candidate gene and its possible effects, effectively simplifying the tangle by only focusing on a few genes and their interactions at a time. Microarray analysis has been widely used in genomic experimentation, as it generates statistical information that can be analysed to create a picture of the likelihood of gene interactions that cause a particular effect. Using these methods, Wang and colleagues cross-referenced five profiles of MDD expression with a statistical model that compensated for any confounding variables. Using meta-analysis statistical models, they demonstrated that the statistical likelihood of the set of MDD candidate genes to cause depression was significantly improved when environmental variables such as sex, age, and ethnicity were taken into account [8]. This suggests that there may be a pairwise link between ethnicity and certain MDD candidate genes, and their likelihood of causing depression symptoms. However, the study did not go into detail about the possibility of an ethnicity/MDD link further than noting the statistical strength of their results, indicating that more research is necessary to reach a conclusion.

Another method of investigation into MDD candidate genes, and perhaps the most relevant to our current study, is the examination of genetic expression in the blood. As Yi et al. demonstrated in 2012, blood is especially useful in determining biomarkers for a particular health condition, as it is the only part of the body that is in constant contact and circulation with multiple tissues, including the brain. Therefore, extraction and analysis of patient DNA from the blood are likely to preserve MDD genes as they are expressed, and may also pick up DNA from tissues and organs being affected by the diseases commonly associated with MDD, allowing a broad picture of how MDD is affecting all areas of the body, and hence its impact on overall patient health [9].

The methods outlined thus far have shown how new techniques and approaches are generating insight into the puzzle of depression's biological and environmental causes. However, the question of which specific biomarkers are most relevant, and which groups of people they effect, must still be addressed. 


\section{ESTABLISHED CANDIDATE GENES AND BIOMARKERS FOR MDD}

As mentioned above, extracting the DNA from components of blood may be an extremely useful tool in diagnosing and tracking depression and its associated conditions. This approach may be applied to several demographic groups, especially those that exhibit other diseases in association with depression. This has led to the discovery of gene variants that are triggered by stressful or traumatic events, and that directly influence a person's resilience against depressive disorders. It is well known that stress and trauma triggers both physiological and psychological responses from the body, governed by a feedback loop of stress-response hormones in the brain. In her 2012 article, Hughes outlines a particular stressmediating gene, FKBP5, that works in governing the brain's responses to stress. Hughes found that people in low socioeconomic demographic groups, who had experienced childhood abuse, a variant of FKBP5 resulted in an increased likelihood of developing disorders such as PTSD and MDD later in life [10].

Another gene variant linked to the development of depressive disorders has been found in adolescents and young adults, furthering the hypothesis that certain demographic groups are more vulnerable to MDD. Working on the established premise that the regulation of neurotransmitter chemicals in the brain plays an important role in regulating mood, it was found that in young adults and adolescents, a particular polymorphism in a serotonin transporter gene, 5-HTTLPR, predicted the development of MDD and mood disorders in adult life [1].

This leads us to further question other possible links between a person's environment and demographic, their subjective stresses, and the development of depressive disorders.

In particular, aging and elderly adults show an association between biomarkers for depression [11] and the progression of neurodegenerative disorders such as dementia and Alzheimer's, as shown by analysis of blood plasma samples [12]. This study found plasma biomarkers, involving specific associated proteins and gene variants, for depression that may be used in future to trace the pathology of other mood-affecting disorders common to the elderly. Interestingly, the gene variants found to be linked to depressive disorders in older adults were also found to be linked to condition affecting the immune system. This suggests that depressive disorders have the ability to affect the disease trajectory of a person's whole life, making it more important to understand a person's genetic vulnerability to MDD. A group of polymorphisms in the VEGF gene was found to elevate in response to depression symptoms in elderly subjects. This is particularly of note due to the VEGF gene's additional association with increased leukocyte expression in depressed patients [12].

Apart from the elderly, prenatal exposure to depression in the mother has also been shown to result in the alteration of genes, leading to different responses to stress and trauma in the infant after birth. In particular, during gestation, foetal exposure to maternal depression was found to be correlated with the methylation of specific receptor genes that govern the hypothalamic-pituitary-adrenal axis system. This HPA system is crucial in mediating human stress responses and cortisol levels. Alteration of receptor genes that govern this axis, therefore, result in differences in cortisol levels in response to stress and trauma, which may result in the development of depressive and anxiety disorders later in life [13]. Infants themselves have also been shown to be more likely to develop depression when exposed to stress and trauma early in life. Early stresses administered to infant mice caused epigenetic alteration of the regulatory regions of the argenine-vasopressin (AVP) gene, which in turn helps govern and regulate the HPA axis [2]. Therefore, it seems clear that environmental exposure to stress in infants is particularly responsible for determining the infant's future responses to stress, and their likelihood to develop depression.

This evidence of environmental determination of genetic expression has been a commonly documented phenomenon in the literature for many years now. One of the first, and perhaps best known, examples of such epigenetic events is the process of methylation, where a region of a specific gene is altered so that it is transcribed differently, giving a different result than originally written. In particular, genes involved in endorphin and neurotransmitter release and regulation in the brain have been shown to be susceptible to environmentally caused methylation, causing differences in neurotransmitter and endorphin levels in the brain. Naturally, this would alter a person's mood and affect considerably, even triggering depression. For example, McGowan and Kato's paper from 2008 demonstrates how environmental methylation caused a variant in the transcription-controlling region of a serotonin transporter gene was correlated with increased neurotic symptoms, including symptoms associated with depression [14]. However, the report was inconclusive as to whether this variant specifically caused depressive symptoms, despite the effect on serotonin transport and regulation.

Direct evidence of the environment's ability to change genetic expression is also seen in the alteration of telomeres in response to environmental stresses [15]. In their 2012 paper, Teyssier et al. reported that telomere functioning was directly related to anxiety and mood, especially in women diagnosed with MDD. Telomeres shorten and degrade in response to environmental stresses, and are responsible for cellular aging. As telomeres shorten, the DNA they house also degrades, providing the potential for differences in expression and transcription. Teyssier et al. were able to isolate two specific genes that usually control the dysfunction of telomeres and cell aging, and found them to be up-regulated in the blood of women suffering depression [16]. This is direct evidence that the environment plays a major role in shaping a person's mental health, and provides a clear causal link between the environment and depression.

The environment as a major factor in determining one's chances of developing MDD is found in yet another study. In their 2007 paper, Ribeiro et al. reported that the brain's structure itself may change in response to genetic variance, leading to increased statistical likelihood of depression. It was found that a polymorphism in the gene for brain-derived 
neurotrophic factor (BDNF) is directly linked to depression in members of a specific ethnic group-one of the first papers to do so. Mexican-American carriers of the homozygous allele of this gene had an increased probability of depression, as the BDNF gene is involved in developing and maintaining neurons in the brain [17]. Although this paper makes a clear link between a specific candidate gene for depression and a particular ethnic group, it must be noted that this is one of the few papers in the literature that confidently makes this claim.

Brain remodelling as a result of stress has also been documented in work by Gounko et al. as recent as 2012, although no distinction is made between the structural change observed and any ethnic group. Rather, the study focused on the effects of stress on the actual structure of the brain, in particular the decrease in neuronal spine populations observed in the hippocampus and prefrontal cortex in response to chronic stress [18]. While this paper provides clear and direct observable evidence of the environment and its stresses being able to physically change a person's brain structure - and by extension their propensity for depression - again there is no link to any particular race.

More recently, a study last year analysed a population of Han Chinese living in the United Kingdom, and found polymorphisms of the dendritic cell nuclear protein DCNP1 within the ethnic group. Haplotype analyses for eight single nucleotide polymorphisms along DCNP1 were analysed, within a framework of meta-analyses of Chinese and Europeans for ethnicity association [19]. No association was found between the polymorphisms found in the Han Chinese population and MDD. However, this study shows the beginnings of a new approach to tackling this field, one more likely to yield definitive results and a start point for unravelling the web of MDD susceptibility: the metaanalysis. By cross-referencing two ethnic groups side by side, any association between ethnicity, the polymorphisms being studied, and MDD itself were able to be ruled out in a single study.

Another study conducted in 2013 focused on the Fars ethnic group in Iran, and found a serotonin transporter polymorphism in those with MDD. The 5-HTTLPR polymorphism affected serotonin reuptake inhibitors, and proved to affect the clinical outcomes of those patients treated with the antidepressant drug Citalopram [20]. This study is notable chiefly in its successful correlation of patient genotype - specifically, a polymorphism found within a particular ethnic group-and the clinical outcome of treatment. However, the study focused on only one particular ethnic group, and so the question of whether the same or similar effects of polymorphisms on clinical outcomes are found within other ethnicities. Here again, we see the need for a meta-analysis to further examine any possible link between ethnicity and the effects of MDD.

Interestingly, work has not only focused on ethnicity, but rather the factors in a patient's life which are more likely to result in a definitive association or causal link. Last year, a team in the USA correlated comorbidity between MDD and marijuana use, due to a polymorphism in the dopamine receptor D4. Notably, the study controlled for not only ethnicity, but other potentially confounding factors such as gender and age [4]. This shows that ethnicity and its resulting peculiarities of genotype can be quite easily overshadowed in the clinical perspective by lifestyle factors. That is, does lifestyle and its myriad factors carry more weight towards MDD susceptibility than ethnicity alone? Once again, the quest to unravel the genetic riddle is met with the central question of epigenetics: is it nature or nurture, that most defines us?

Most recently, a 2014 study identified oxidative and inflammatory stress biomarkers as sharing a peripheral role in indicating MDD, paving the way for further molecular work and supporting the role of environmental stress as a key factor in the disease's prevalence [21]. At the same time, MDD was identified as a risk factor for cases of cancer, indicating there is much yet to be understood of MDD's multivariate role in a patient's overall health [22]

From the research at hand, it is clear that the problem of MDD susceptibility, while clearly genetic, has far too many contributing and potentially confounding factors to be tackled by studies that work independently. A more longterm, multi-faceted approach is indicated. In Europe, the HELIUS study started in 2013 aims to tackle the physical and socioeconomic factors affecting the risk of different diseases, and to analyse the different health risks associated with the many ethnic groups living in Europe [23]. Designed to be multi-generational, HELIUS establishes a precedent for further research, acknowledging that while some diseases do have a clear ethnicity association (for example, haemoglobinopathies), an ethnicity link is far less likely for a complex disease such as MDD. Again, it is clear we must account for the effects of epigenetics. Nevertheless, studies such as HELIUS are good examples of how this research must continue in future.

\section{DISCUSSION}

From this, the question of which specific groups of people, if any, are most affected by the different depression candidate genes is raised. It appears clear from the work discussed above that, above all, it is one's environment, not one's ethnicity that determines the likelihood of developing mood disorders such as MDD. Chronic stress, particularly stress associated with living in built-up areas and with earlylife trauma, appears to be the leading physical causal factor for developing depression. Chronic stress and trauma has the documented ability to change not only the expression of genes that are responsible for maintaining mood and hormonal stress responses, but the physical structure of the brain, making it a clear culprit for the depression affecting millions of people worldwide.

Apart from the work of Ribeiro et al. discussing the genetic variant found in Mexican-Americans, the majority of the literature finds no link or association between ethnicity and the probability of depressive disorders. From the literature, it appears quite clear that the type of environment people are subjected to plays a key role in shaping the genetic expression and brain structure linked with depression. Although one candidate gene was found to be ethnically linked [19], the appearance of this gene in only one paper makes it impossible to rule out the environment as a causal factor of the gene variant, rather than ethnicity itself. 
Furthermore, while certain ethnicities do show particular polymorphisms that influence clinical outcome and comorbidity with other lifestyle factors, it is impossible to further analyse any possible links without further study.

\section{SUGGESTIONS FOR FURTHER RESEARCH}

Building on these findings, further research could aim to re-examine both specific socioeconomic groups commonly associated with depression and any ethnic groups with depression candidate genes, and cross-reference these associations to determine which factor is the cause of the variant. To settle the question of environment $v s$. ethnicity, more studies are needed that specifically examine the statistical strength of each factor. However, the current research indicates that environment is the chief causal factor of depression in populations, rather than ethnicity. Moreover, it appears highly likely that the heritability associated with depression comes from the genetic changes wrought by the environment, providing further evidence for the environment's key role in shaping the pathogenesis of depression. Taking this into account, meta-analyses of both ethnicity and the lifestyle factors (both socioeconomic and environmental) that are seen within that ethnic group in particular regions of the world could shed further light on whether we are looking at a cut-and-dry case of nature $v s$. nurture, or a more complex interaction of the two. In short, understanding MDD will require studies in future that acknowledge complex factors for a complex disease.

\section{CONFLICT OF INTEREST}

The authors confirm that this article content has no conflict of interest.

\section{ACKNOWLEDGEMENTS}

Declared none.

\section{REFERENCES}

Hankin B. Future directions in vulnerability to depression among youth: Integrating risk factors and processes across multiple levels of analysis. J Clin Child Adoles Psychol 2012; 41(5): 695-718.

[2] Murgatroyd C, Wu Y, Bockmuhl Y, Spengler, D. Genes learn from stress: How infantile trauma programs us for depression. Epigenetics 2010; 5(3): 194-9.

[3] Nestler, E. Stress makes its molecular mark. Nature 2012; 490 (7419): 171-2

[4] Bobadilla L, Vaske J, Asberg K. Dopamine receptor (D4) polymorphism is related to comorbidity between marijuana use and depression. Addict Behav 2013; 38: 2555-62.
[5] Chong S, Abdin E, Vaingankar J, et al. Subramaniam M. A population-based survey of mental disorders in Singapore. Ann Acad Med Singapore 2012; 41: 49-66.

[6] Melchior M, Chastang J-F, Head J, et al. Socioeconomic position predicts long-term depression trajectory: A 13-year follow-up of the GAZEL cohort study. Mol Psychol 2013; 18: 112-21.

[7] Jia P, Kao C-F, Kuo P-H, Zhao Z. A comprehensive network and pathway analysis of candidate genes in major depressive disorder. BMC Syst Biol 2011; 5(3): S12.

[8] Wang X, Lin Y, Song C, Sibille E, Tseng G. Detecting diseaseassociated genes with confounding variable adjustment and the impact on genomic meta-analysis: With application to major depressive disorder. BMC Syst Biol 2012; 13: 52 .

[9] Yi Z, Li Z, Yu S, et al. Blood-based gene expression profiles models for classification of subsyndromal symptomatic depression and major depressive disorder. PLoS ONE 2012; 7(2): e31283.

[10] Hughes V. Roots of resilience. Nature 2012; 490: 165-7.

[11] Wolkowitz O, Reus VI, Mellon SH. Of sound mind and body: Depression, disease, and accelerated aging. Dial Clin Neurosci 2011; 13(1): 25-39.

[12] Arnold SE, Xie SX, Wang L-S, et al. Plasma biomarkers of depressive symptoms in older adults. Transl Psychiatry 2012; 2 : e65.

[13] Oberlander T, Weinberg J, Papsdorf M, et al. Prenatal exposure to maternal depression, neonatal methylation of human glucocorticoid receptor gene (NR3C1) and infant cortisol stress responses. Epigenetics 2008; 3(2): 97-106.

[14] McGowan P, Kato T. Epigenetics in mood disorders. Environ Health Prev Med 2008; 13: 16-24.

[15] Blackburn E, Epel E. Too toxic to ignore. Nature 2012; 490: 16971.

[16] Teyssier J-R, Chauvet-Gelinier JC, Ragot S, Bonin B. Upregulation of leucocytes genes implicated in telomere dysfunction and cellular senescence correlates with depression and anxiety severity scores. PLoS ONE 2012; 7(11): e49677.

[17] Ribeiro L, Busnello J, Cantor $\mathrm{R}$, et al. The brain-derived neutortrophic factor rs6265 (Val66Met) polymorphism and depression in Mexican-Americans. Neuroreport 2007; 18(12): 1291-3.

[18] Gounko N, Swinny J, Kallcharan D, et al. Corticotropin-releasing factor and urocortin regulate spine and synapse formation: Structural basis for stress-induced neuronal remodelling and pathology. Mol Psychol 2013; 18: 86-92.

[19] Yi H, Wang Y-J, Hua L, et al. Lack of association between dendritic cell nuclear protein-1 gene and major depressive disorder in the Han Chinese population. Prog Neuro-Psych Bio Psych 2013; 45: 7-10

[20] Sahraian S, Babashams M, Reza-Soltani P, Najmabadi H, Kahrizi K, Gorgani S. Serotonin transporter polymorphism (5-HTTLPR) and Citalopram effectiveness in Iranian patients with major depressive disorder. Iranian J Psychol 2013; 8(2): 86-91.

[21] Lopresti A, Maker G, Hood S, Drummond P. A review of peripheral biomarkers in major depression: The potential of inflammatory and oxidative stress biomarkers. Prog Neuro-Psych Bio Psychol 2014; 48: 102-11.

[22] Currier M, Nemeroff C. Depression as a risk factor for cancer: From pathophysiological advances to treatment implications. Ann Rev Med 2014; 65: 203-21.

[23] Stronks K, Snijder M, Peters R, Prins M, Schene A, Zwinderman A. Unravelling the impact of ethnicity on health in Europe: The HELIUS study. BMC Public Health 2013; 13: 402. 